\title{
(2) Does cryoneurolysis result in persistent motor OPEN ACCESS deficits? A controlled study using a rat peroneal nerve injury model
}

\author{
Sameer B Shah, ${ }^{1}$ Shannon Bremner, ${ }^{1}$ Mary Esparza, ${ }^{1}$ Shanelle Dorn, ${ }^{1}$ \\ Elisabeth Orozco, ${ }^{1}$ Cameron Haghshenas, ${ }^{1}$ Brian M Ilfeld (D) , ${ }^{2}$ Rodney A Gabriel (D) , \\ Samuel Ward ${ }^{1}$
}

1 Orthopedic Surgery, University of California, San Diego, La Jolla, California, USA

${ }^{2}$ Anesthesiology, University of California, San Diego, La Jolla, California, USA

Correspondence to Dr Brian M llfeld, Anesthesiology, University of California, San Diego, La Jolla, CA 92093, USA:

bilfeld@ucsd.edu

Received 11 November 2019 Revised 13 January 2020 Accepted 14 January 2020 Published Online First 29 January 2020

\section{man} chronic pain states. However, it remains unclear whether persistent functional motor deficits remain following cryoneurolysis of mixed sensorimotor peripheral nerves, greatly limiting clinical application of this modality. To help inform future research, we used a rat peroneal nerve injury model to evaluate if cryoneurolysis results in persistent deficits in motor function.

Methods Male Lewis rats $(n=30)$ had their common peroneal nerves exposed bilaterally at the proximal lateral margin of the knee and subsequently underwent cryoneurolysis on one limb and sham treatment on the contralateral limb. Outcomes were evaluated on days 3 , 14, 30, 90 and 180. The primary end point was motor function, based on ankle dorsiflexion torque. In addition, sensory function was tested based on von Frey's filament sensitivity to the peroneal sensory distribution. A subset of animals was sacrificed following functional testing at each time point, and general tissue morphology, connective tissue deposition, and axon counts were evaluated.

Results Motor deficits in treated limbs were observed at 3 and 14 days but had resolved at time points beyond 1 month. Bilateral sensory deficits were also observed at 3 and 14 days, and also resolved within 1 month. Consistent with motor functional deficits, axon counts trended lower in treated nerves compared with contralateral controls at 3 days; however, axon counts were not significantly different at later time points. Conclusions When applied to a mixed sensorimotor nerve, cryoneurolysis did not result in persistent motor deficits.

\section{INTRODUCTION}

Pain following surgery is frequently treated with local anesthetic-based peripheral nerve blocks to improve analgesia, decrease/negate opioid reliance, and decrease the incidence/severity of persistent postsurgical pain. However, the duration of surgical pain is often far longer than the duration of even the longest-acting single-injection peripheral nerve block; and, perineural local anesthetic infusion is limited to less than 1 week in most cases. ${ }^{1}$
Cryoneurolysis is a non-opioid analgesic modality used to treat both acute and chronic pain involving the application of extremely low temperatures to a peripheral nerve to induce Wallerian degeneration and consequent abrogation of nerve conduction. ${ }^{2}$ Axons regenerate along the undisturbed epineurium, perineurium, and endoneurium from the site of ablation towards their distal targets at about $1-2 \mathrm{~mm} / \mathrm{day}^{3}{ }^{3}$ resulting in a nerve block with a variable duration, but typically measured in multiple weeks. ${ }^{4}$ While cryoanalgesia has been used for decades to treat primarily chronic pain, ${ }^{4-8}$ the advent of percutaneous cannulas and ultrasound guidance dramatically increases the potential applications for its use in treating acute pain as well. ${ }^{9-11}$

To date, most published research involves the functional and histological recovery of sensory nerves ${ }^{12-16}$; however, mixed motor-sensory nerves may also be targeted. ${ }^{4} 101718$ Because cryoneurolysis affects both sensory and motor neurons, concern exists regarding residual and persistent motor weakness due to either incomplete regeneration or motor unit clustering. Given that interest in cryoneurolysis is increasing due to an increased awareness of the pervasiveness of inadequately-controlled postoperative pain, ${ }^{19}$ current public health efforts to decrease opioid use, ${ }^{20}$ and recognition that chronic, persistent postoperative pain is associated with inadequately-controlled perioperative pain, ${ }^{21}$ it is imperative that any long-term cryo-induced motor deficits be identified.

In this controlled study, we used a sensitive and validated rat common peroneal (fibular) nerve injury model to evaluate if cryoneurolysis results in persistent motor function deficits, based on recovery of ankle dorsiflexion torque.

\section{METHODS}

\section{Study intervention}

Thirty $300 \mathrm{~g}$ male Lewis rats underwent survival surgery under isoflurane anaesthesia with analgesia provided with preoperative subcutaneous injection of $0.3 \mathrm{mg}$ sustained release buprenorphine (Buprenorphine SR-LAB, ZooPharm, Windsor, Colorado, USA). Common peroneal (fibular) nerves were exposed bilaterally at the proximal lateral margin of the knee joint using a technique previously described. $^{22} 23$ The fascial sheath overlying the nerve was left intact. On the left limb, cryoneurolysis was performed with a console based 
clinical cryoneurolysis machine (PainBlocker, Epimed International, Farmers Branch, Texas, USA). An $18 \mathrm{G}$ trocar with a tapering tip was positioned immediately adjacent to the target nerve and three $2 \mathrm{~min}$ cryoneurolysis cycles separated by $1 \mathrm{~min}$ thaw periods were applied using nitrous oxide. This protocol is a typical clinical protocol, ${ }^{3}$ and given the size and exposure of the rat peroneal nerve, may be viewed as aggressive. On the right limb, sham cryoneurolysis was performed, with exposure, tip contact, and time of contact as above, but no freezing.

Multiple criteria were considered to retain consistency within our cryoneurolysis protocol. Frosting of the cannula tip as well as formation of an ice ball encompassing the nerve was confirmed for each cycle. Repeatability in freezing technique was confirmed based on: (i) consistent exposure of the peroneal nerve; (ii) device indicator confirming sufficiently cooled cannula tip; (iii) frosting of cannula; (iv) visual verification of contact of cannula tip directly on fascial sheath overlying peroneal nerve; (v) ice ball formation on tissue surrounding nerve; (vi) fasciculations on at least one of the freeze cycles. Increased local blood flow was typical during a freeze cycle. Cannulas were thawed completely before detachment from tissue to avoid imposing mechanical force on the nerve by cannula manipulation.

On the contralateral limb, the sham (control) treatment was administered by positioning the cryoablation cannula immediately adjacent to the target nerve for the same three 2 min on, 1 min off pattern as before, but without freezing (ie, no nitrous oxide). Overlying muscles were repaired by closing the fascia with 4-0 polyglycolic acid (PGA) sutures, and wound clips were used to close the skin incision.

\section{Outcome measures}

End points were assessed at 3, 14, 30, 90, and 180 days following treatment, a time frame over $3 \mathrm{x}$ longer than that during which a small population of persistent motor deficits were observed. ${ }^{23}$ Motor function was assessed bilaterally via isometric ankle dorsiflexion torque, using a previously-described and validated method. ${ }^{23}$ Briefly, following anaesthesia induction ( $2 \%$ isoflurane, $2.0 \mathrm{~L} / \mathrm{min}$ ), dorsiflexors were activated via the common fibular nerve using a nerve cuff $(15 \mathrm{~V}$ at $100 \mathrm{~Hz}, 0.3$ $\mathrm{ms}$ pulse duration and $650 \mathrm{~ms}$ train duration), while torque was measured using a custom-designed dynamometer. ${ }^{22}$ The dynamometer was created from a dual-mode servo motor (Aurora Model 305B, Aurora Scientific, Aurora, Ontario, Canada) which has a force resolution of $1 \mathrm{mN}$, a linearity of $99.8 \%$ and a step response time under $2 \mathrm{~ms}$. Peak torque was defined as the absolute peak isometric torque elicited after the onset of stimulation. Sensory testing was performed bilaterally with von Frey filaments applied to the dorsal surface of the foot (peroneal nerve sensory distribution). ${ }^{24}$ Rats were acclimated with one round of practice testing preoperatively. Rats were required to plant their entire foot (heel to toe) prior to testing, and paw withdrawal pattern was assessed using the up-down method. ${ }^{25}$ Data are represented as the difference in post-treatment sensitivity (ie, force eliciting withdrawal) minus baseline sensitivity, with more negative values indicating hypersensitivity.

\section{Histological outcomes}

A subset of animals was sacrificed under anaesthesia following functional testing at each time point via intracardiac injection of pentobarbital sodium and phenytoin sodium $(150 \mathrm{mg} / \mathrm{kg})$. In addition, three animals were euthanised immediately after freezing, for histological analysis of acute structural changes resulting from cryoneurolysis. For each animal, the common peroneal nerve was harvested bilaterally, and snap frozen using liquid nitrogen cooled isopentane. ${ }^{26}$ Transverse sections of the nerve underlying the distal edge of the cryoablation site and axial sections of tibialis anterior (TA) muscle $\sim 5 \mathrm{~mm}$ distal to its insertion at the knee were taken per previously published methods, and slides were stored at $-80^{\circ} \mathrm{C}$ until use. ${ }^{23}{ }^{26}$ Sections were stained with H\&E, Masson's trichrome (Polysciences, Warrington, Pennsylvania, USA), Wheat Germ Agglutinin (WGA) conjugated to AlexaFLuor 594, DAPI, and/or $\beta 3$-tubulin+laminin. The neuron-specific $\beta 3$-tubulin was used as an axonal marker for axon counting, laminin provided an outline for delineating distinct nerve fibers, and WGA outlined muscle fibers. Sections were permeabilised with $0.2 \%$ Triton-X, blocked with blocking buffer (10\% goat serum, 3\% bovine serum albumin (BSA) in phophate buffered saline (PBS)) then incubated with primary antibodies mouse anti- $\beta 3$-tubulin (Sigma-Aldrich; $1: 500$ dilution) and rabbit anti-laminin (Sigma-Aldrich; 1:1000 dilution) for 1 hour. Slides were then incubated with AlexaFluor 488 goat-anti-mouse and AlexaFluor 594 goat-anti-rabbit secondary antibodies, then coverslipped with Vectashield Hardset with 4',6-diamidino-2-phenylindole (DAPI ;Vector Labs, Burlingame, California, USA).

Fluorescent images were acquired using a Leica DM6000 microscope (Leica Microsystems, Buffalo Grove, Illinois, USA) equipped with a Leica DFC365 FX camera (Leica Microsystems) using a $10 \mathrm{X}$ or a $63 \mathrm{X}$ oil immersion objective, and brightfield images were acquired at 20X using a Leica SCN400 slide scanner (Leica Microsystems). Axon counts were estimated from two sections, based on manual counting of $\beta 3$-tubulin-positive puncta in selected $63 \mathrm{X}$ images covering at least $35 \%$ of the nerve cross-sectional area, and subsequent scaling based on cumulative fascicular area measured from $10 \mathrm{X}$ images. ${ }^{26}$ This stereological sampling approach procedure provided sufficient coverage to provide confidence in axon counts, while also enabling the inclusion of sections with minor tears or regions of inconsistent staining. All histological analysis was blinded to the data analyst.

\section{Statistics}

The primary null hypothesis was that a clinically-relevant cryoneurolysis protocol does not induce persistent motor functional deficits. This hypothesis was tested based on measurement of ankle dorsiflexion torque as a primary end point. We achieved statistical power of 0.86 for $n=6 /$ group, for a moderate effect size of 0.5 and alpha $=0.05$. Secondary null hypotheses were that a clinically-relevant cryoneurolysis protocol did not induce persistent mechanical sensory functional deficits or structural changes, as measured by von Frey's hair testing and comparison of axon counts, respectively. Parametric statistical testing was performed for all comparisons. Normality was confirmed using the Shapiro-Wilk normality test. Mean motor, sensory and structural outcomes were compared using mixed design 2-way analysis of variance (ANOVA), which examined main and interaction effects of time (unpaired factor) and intervention (paired factor). Tukey's Honestly Significant Difference (HSD) post-hoc test was used to compared individual means and accounted for multiple comparisons. Prism 8 (GraphPad, La Jolla, California, USA) was used for all statistical testing.

\section{RESULTS}

As determined by our six criteria, consistent freezing was confirmed for each experiment. General histological assessment using H\&E showed clear changes to tissue surrounding the epineurium immediately following cryoneurolysis, including 


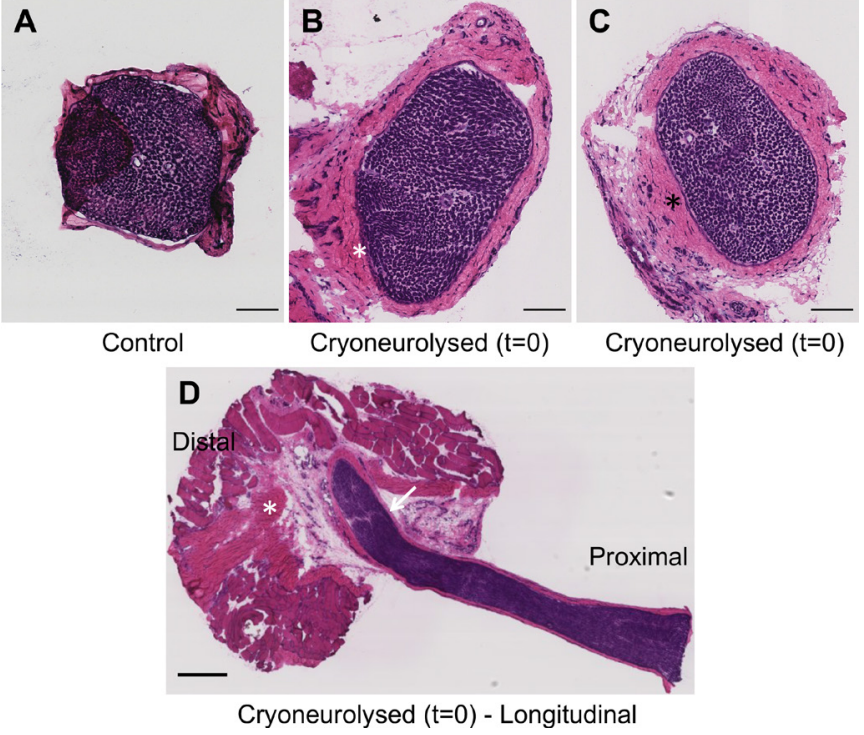

Figure 1 Cryoneurolysis results in immediate tissue changes exterior to, but not within, the ablated nerve (H\&E label). (A) Control nerve in cross-section. Note scale of comparatively thin epineurium. Some tissue has folded over to the left side of the image. (B and C) Crosssections of two different nerves in region of cryo-ablation. Note clear capsule formation around epineurium (asterisk *), and connectivity to underlying muscle tissue following ablation protocol. Bars 100 um. (D) Longitudinal section of nerve in region of cryo-ablation. Note seemingly normal nerve tissue within the epineurium, but apparent freeze damage and connectivity to underlying muscle outside of the nerve (asterisk *). The 'crack' in the nerve (arrow) is likely to be an artifact of sectioning. Bar $100 \mu \mathrm{m}$.

thickening of the epineurial wall and adhesion to surrounding musculature (figure 1A-D). However, the interior of the nerve appeared grossly intact. Consistent with these observations, trichrome staining in cryoneurolysed-but not sham-nerves indicated connective tissue deposition around the nerve, with structurally intact interiors (figure 2). These changes persisted throughout the evaluation period (figure 2D). Axonal localisation within nerves also appeared qualitatively normal, in all groups (figure 3A-D). At 3 days after surgery, but at no other time point, decreased axon counts (indicative of Wallerian degeneration) were observed in cryoneurolysed nerves compared with sham (figure 3E). No pain-associated behavioral changes were observed after surgery at any time point.

\section{Motor function}

To test the primary hypothesis that cryoneurolysis did not result in persistent motor deficits, ankle dorsiflexion torque was evaluated. Two-way ANOVA showed main effects of both time $(\mathrm{p}<0.001)$ and treatment $(\mathrm{p}<0.05)$ on peak dorsiflexion torque, but no interaction effect. Consistent with animal growth, motor function gradually improved in this group over the 180-day evaluation period. Sham cryoneurolysis did not result in motor impairment at any time point, validating that surgical approach did not affect the control group (figure 4A). Post hoc testing of individual means revealed that peak torque was not significantly different between cryoneurolysis and sham groups at any time point (figure 4A). Recovery of torque to statistically insignificant magnitudes was observed at 30 days $(\mathrm{p}=0.88)$; and, in support of the primary hypothesis, torque was indistinguishable between treatment and control limbs at 3 and 6 months $(p>0.99$,

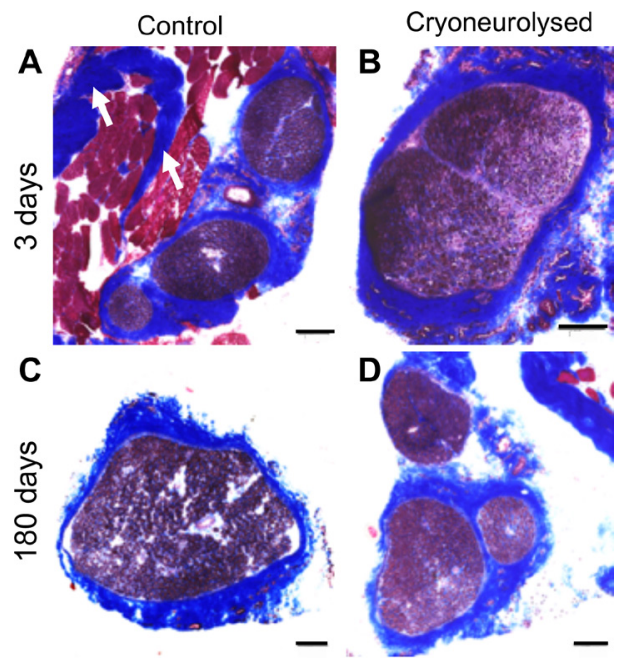

Figure 2 Cryoneurolysis results in persistent tissue changes exterior to, but not within, the ablated nerve (trichrome label). (A-D) Sample trichrome labeling for sham $(A, C)$ and cryoneurolysed $(B, D)$ nerve at 3 days $(A-B)$ and 180 days $(C-D)$. Note more integration of frozen nerves with surrounding nerve bed, in contrast to sharper epineurial borders in sham-treated nerves. White arrows in (A) indicate muscle connective tissue, which is distinct from epineurial tissue. Bars $=100 \mu \mathrm{m}$. Note that fasciculation patterns appear different in each section; this reflects anatomical variation in internal branching from nerve to nerve, despite consistency in section location.

figure 4A). Consistent with these observations, no differences in fiber morphology, spacing between muscle fibers, or centralized nuclei were observed between sham and contralateral TA muscle 6 months after injury (figure 4B-E).

\section{Sensory function}

Two-way ANOVA revealed main effects of time $(\mathrm{p}<0.001)$, but not treatment $(\mathrm{p}=0.45)$ on mechanical sensitivity in the dorsal paw (peroneal sensory distribution). Post-hoc testing revealed no statistically significant difference between treated and control limbs at any time point (figure 5), with slight (1-2 filament) bilateral hypersensitivity at Day 3, possibly associated with surgical trauma. All hypersensitivity resolved by 1 month, and normal sensitivity was maintained at 3 and 6 months (figure 5).

\section{DISCUSSION}

This study provides quantitative evidence in an animal model that cryoneurolysis does not result in persistent functional deficits when applied to a mixed sensorimotor nerve of a rat. The expected motor deficits existing within the first two posttreatment weeks (days 3 and 14) resolved by 1 month, mirroring the histological evidence of Wallerian degeneration followed by regeneration. While we cannot extrapolate the timing of motor recovery in laboratory animals to a clinical setting, these results are reassuring in that cryoneurolysis, a modality that is approved by the United States Food and Drug Administration and used clinically for multiple decades-does not appear to induce persistent deficits.

Our data should be placed in the context of several previous studies that examined effects of cryoneurolysis on nerve structure and neuromuscular function. Whittaker published a comprehensive assessment of nerve morphology and ultrastructure after freezing the ventral epithelium of the golden hamster tongue. ${ }^{27}$ Significant disruption of myelination as well as 

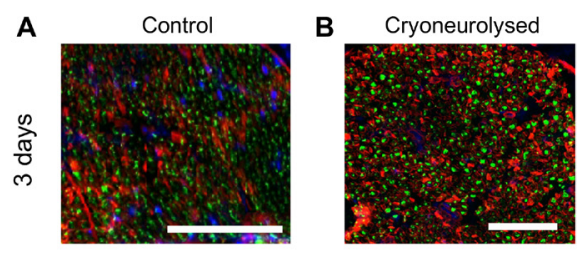

C
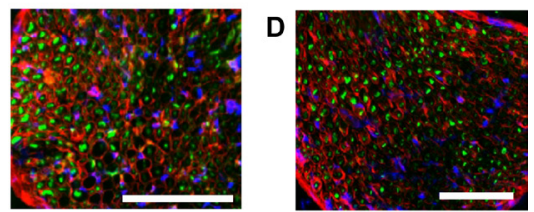

E

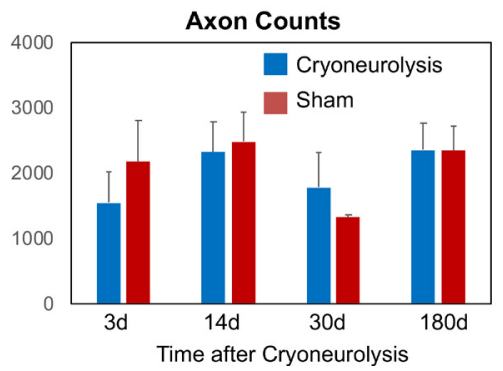

Figure 3 Nerve morphology and axon counts are not affected by cryoneurolysis. (A-D) Sample B3-tubulin (axons, green), laminin (fiber borders, red), and DAPI (nuclei, blue) labeling for sham $(A, C)$ and cryoneurolysed $(B, D)$ nerve at 3 days $(A-B)$ and 180 days $(C-D)$. Bars $=100 \mu \mathrm{m}$. (E) Quantification of axon counts (mean \pm SEM) shows no effect of time or treatment, and NO statistically significant difference in axon number (sham vs cryoneurolysis) at any time point (mixed-design two-way ANOVA and Tukey's HSD post-hoc testing). A trend towards fewer axons at 3 days, but no other time point, is observed, consistent with motor recovery. Bars $=100 \mu \mathrm{m}$.

signatures of axon degeneration were observed within an hour of freezing, with substantial myelin disruption and absorption as well as axolemmal disappearance. By day 14, early signs of regeneration, including axonal regrowth within early myelinating sheaths, were apparent (although functional testing was not performed). While we did not perform electron microscopy on ablated nerves, we observed structural damage to the nerve at 3 and 14-day time points, although not as comprehensive as in the lingual nerve model. Our timeline of functional recovery by 1 month also suggested structural regeneration at an earlier time point; however, differences in species and scale preclude a direct comparison.

Deleo, Wagner and colleagues published a subsequent series of studies on outcomes following cryoneurolysis of the rat sciatic nerve with the intent of validating a new model of pain. ${ }^{29-33}$ In these studies-as for our investigation-adverse sensory outcomes were observed in both the cryo-treated and control limbs. ${ }^{34}$ However, sensory deficits persisted beyond 28 days suggesting more severe damage, in contrast to our observations of sensory recovery by 1 month. The authors speculated that the persistence of bilateral deficits was indicative of a central nervous system contribution to neuropathy. ${ }^{34}$ This persistence may also have a methodological basis: in addition to freeze damage, the nerve was decompressed and raised from its bed during cryoneurolyis which may have created a non-physiological heat (cold)-transfer and mechanical environment more conducive to damage (Wagner, personal communication 2019).

A third set of studies by Hsu and Stevenson directly tested motor functional recovery. ${ }^{35} 36$ In this case, a tibial nerve injury
A
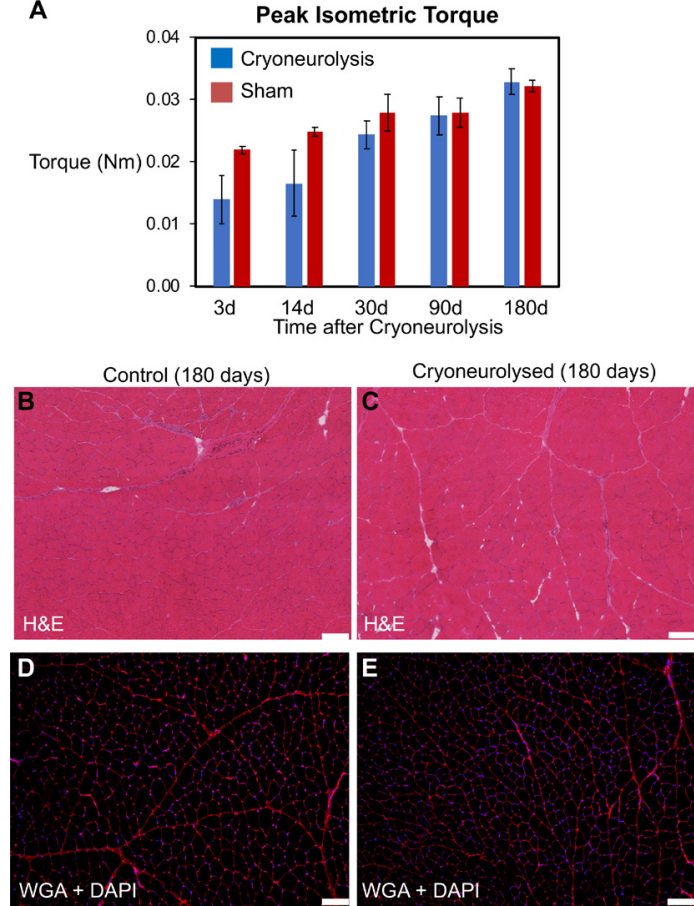

Figure 4 Motor function and muscle structure are not persistently affected by cryoneurolysis. (A) Peak isometric torque revealed ipsilateral motor deficits on the ipsilateral (ablated) side at early time points, which resolved by 30 days (main effects of time $(p<0.001)$ and treatment $(p<0.05)$ based on mixed-design two-way ANOVA. No significant differences between individual means (sham vs cryoneurolysis) at any time point based on Tukey's HSD post-hoc testing). Values are provided as mean \pm SEM. (B) Control and (C) cryoneurolysed TA muscle at 180 days show no differences in gross morphology (H\&E labeling). (D) Control and (D) cryoneurolysed TA muscle at 180 days show no differences in muscle fiber morphology, no widening of intra-fiber spacing, and NO centralised nuclei (WGA and DAPI labeling). Bars $=100 \mu \mathrm{m}$. TA, tibialis anterior; WGA, Wheat Germ Agglutinin.

was created using a single $1 \mathrm{~min}$ long cryoneurolysis application (which in one study was repeated three times at 2-month intervals). Motor function was evaluated using toe spreading

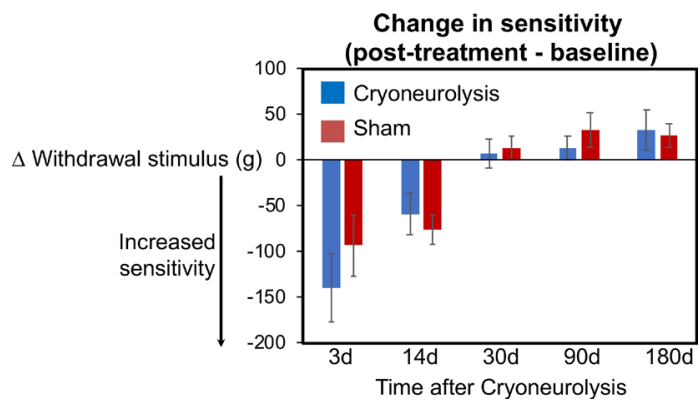

Figure 5 Mechanical sensitivity is not persistently affected by cryoneurolysis. von Frey's testing revealed slight (difference of 1-2 filaments) bilateral increases in sensitivity in the peroneal sensory distribution (dorsal aspect of the paw) at early time points, which resolved by 30 days. (main effects of time $(p<0.001)$ but not treatment $(p=0.45)$ based on mixed-design two-way ANOVA. No significant differences between individual means (sham vs cryoneurolysis) at any time point based on Tukey's HSD post-hoc testing). Values are provided as mean \pm SEM 
and a semi-quantitative walking scale, and reversibility of motor deficits was observed within 50 days. While these results were promising, there were several important limitations to this work that precluded a definitive conclusion. Importantly, a relatively benign cryoablation protocol was used, which may not represent a clinically-typical and more invasive multi-cycle cryoneurolysis protocol. ${ }^{4}$ Most critically, motor functional assessments were semi-quantitative and low in sensitivity, and minimal quantitative assessment of nerve morphology was performed. These confounding factors were compounded by the decision to treat the tibial nerve: while this nerve indeed plays an important role in ankle plantar-flexion and toe abduction, it does not innervate toe extensors and ankle dorsi-flexors which are essential for proper stepping during walking and the uncurling of toes. Thus, their reported semi-quantitative motor score which incorporated qualitative assessments of both gait and toe curling, may not have accurately or adequately described the impacts of their particular injury model. Lastly, a small (though not statistically significant) cohort of animals displayed continued motor deficits at the latest time point. ${ }^{35} 36$ These observations suggested that higher-resolution measurements may be needed to detect more subtle-but persistent-motor deficits. It was for this reason that we undertook the current study using a far more sensitive model of functional motor deficits. Our more sensitive and quantitative functional motor outcomes were qualitatively consistent with these prior studies in that motor deficits were indeed reversible.

Our peroneal nerve injury model and measurement of dorsiflexion joint torque, which have been validated in multiple studies perturbing various aspects of neuromuscular function, 22233738 offer several advantages to prior studies, and provide additional confidence in interpretation of our results. Peroneal nerve injury stands in contrast to sciatic nerve injury, in which both the tibial and peroneal branches are affected. Without a more sensitive outcome specific to each branch, it is difficult to confidently interpret outcomes. For example, following sciatic nerve injury, poor functional recovery may be a result of incomplete or imbalanced regeneration into the two branches. In our model, we injured only one branch, and the corresponding sensory and motor outcomes tested were directly related to the peroneal distribution-that is, joint dorsiflexion torque and sensitivity of the dorsal surface of the foot. Thus, there is no ambiguity, compared with observational assessments of motor function or behavioral outcomes such as Rotarod or grip testing. In addition, we applied a protocol of multiple freeze cycles separated by shorter thaw periods which better-represents a typical clinical application protocol that is intended to provide analgesia and not induce pain. Additionally, the nerve was left attached and unperturbed in its underlying tissue (ie, not decompressed), simulating the clinical reality of percutaneous cryoneurolysis. Thus, we are confident that the reversibility of sensory and motor deficits is not artifactual and is specific to the nature of the nerve injury.

The transient and minor nature $(<2$ von Frey hair's difference) of bilateral sensory hypersensitivity on both the treated and untreated limb may simply reflect discomfort due to bilateral surgical trauma, which may also contribute to the higher variability in motor and sensory function at the 3-day time point (figure 5). However, bilateral sensory hypersensitivity has also been noted in a variety of unilateral nerve injury models, including nerve freezing. ${ }^{34} 3940$ A number of hypotheses have been posed to explain these phenomena, including systemic factors ${ }^{41}$ and central processing of pain. ${ }^{41-43}$ The current study design could not distinguish among these possibilities; as the primary outcome of this study was to evaluate motor function, an important extension of this work is the need for deeper evaluation of sensory outcomes.
Regarding motor deficits, we observed variable muscle weakness ranging from normal to complete loss of function in the active treatment limbs. This may reflect variable and/or incomplete nerve injury and is consistent with both histological and clinical observations. Structural changes to the nerve bed were visible immediately after cryoneurolysis and persisted throughout the 6 month recovery period. However, the degree and rapidity with which surrounding tissue thickened after the initial cycle of cryoneurolysis may impact the delivery of consistent freeze damage within the nerve in subsequent freeze cycles. ${ }^{4}$ Consistent with functional loss at 3 and 14 days, axon counts generally trended downward at 3 days and recovered within 1 month; however, the variability in axonal reduction was also substantial. A vascular contribution to functional deficits has also been proposed, ${ }^{4}$ although such a mechanism is not intuitively apparent from our study. However, our observation of mesoneurial deposition of connective tissue (figure 3) may contribute to entrapment at longer time points, which may result in neurovascular compromise. ${ }^{4445}$

\section{Limitations}

Due to potentially confounding effects of surgical exposure required for high-sensitivity functional testing, we were unable to follow the functional recovery of a single rat longitudinally. Thus, we relied on comparisons to contralateral sham controls, in which there may be some inherent baseline differences in structure and function. ${ }^{46}{ }^{47}$ We powered our study to evaluate the primary outcome of motor function and, in this effort, we were successful. However, this sample size was likely underpowered for more subtle structural outcomes, including distinguishing between sensory and motor-specific histological outcomes. We also acknowledge that cryoneurolysis was not superposed on a model of pain, and so we could not evaluate its impact on pain relief. Finally, while modern clinical deployment of cryoneurolysis is frequently ultrasound guided and percutaneous, we acknowledge that the peroneal nerve was exposed during cryoneurolysis, an approach more commonly performed by surgeons in earlier studies. ${ }^{5}$ Percutaneous insertion of currently-available cryo-delivery cannulas would have been more challenging at the small scale of rat limbs; our technique enabled more consistent placement of the cannula on its target, providing us more confidence in our subsequent results. This approach may also be viewed as more conservative, as no motor deficits were observed in spite of the more invasive approach on a very small nerve target.

\section{CONCLUSIONS}

We combined a typical clinical cryoneurolysis protocol and a sensitive, validated rat peroneal nerve injury model to assess the effect duration of cryoneurolysis on motor function. When applied to a mixed sensorimotor nerve, cryoneurolysis did not result in persistent functional deficits.

\section{Summary statement}

When applied to a mixed sensory-motor nerve in Lewis rats, cryoneurolysis did not result in persistent structural or functional motor deficits.

Acknowledgements The authors appreciate the invaluable assistance of Rochelle Wagner, PhD (Senior Medical Science Liaison, TerSera Therapeutics, Lake Forest, Illinois) for her help in designing the protocol and manuscript review.

Contributors SS: Planning, design, conduct, acquisition of data, data analysis and interpretation, and manuscript preparation. SB, ME, SD, EO, CH: Conduct, acquisition of data and manuscript revision. BMl: Conception, planning, design, data interpretation and manuscript preparation. RAG: Planning, design, conduct, data 
interpretation and manuscript revision. SW: Conception, planning, design, conduct, data interpretation and manuscript revision.

Funding Funding and equipment for this project provided by Epimed International (Farmers Branch, Texas).

Competing interests SS: The following companies have provided material and/ or funding for his research directly to SS's institution: Epimed International (Farmers Branch, Texas). BMI: The following companies have provided material and/or funding for his research directly to BMI's institution: Epimed International (Farmers Branch, Texas); Myoscience (Fremont, California); SPR Therapeutics (Cleveland, Ohio); Infutronics (Natick, Massachusetts); Ferrosan Medical (Szczecin, Poland); and Heron Pharmaceuticals (San Diego, California). RAG: The following companies have provided material and/or funding for his research directly to RAG's institution: Epimed International (Farmers Branch, Texas); Myoscience (Fremont, California); SPR Therapeutics (Cleveland, Ohio); Infutronics (Natick, Massachusetts); and Ferrosan Medical (Szczecin, Poland). SW: The following companies have provided material and/or funding for his research directly to SW's institution: Epimed International (Farmers Branch, Texas); DelNova Inc (Chicago, IL).

Patient consent for publication Not required.

Ethics approval All animal work was prospectively approved by the University of California, San Diego Institutional Animal Care and Use Committee (La Jolla, California).

Provenance and peer review Not commissioned; externally peer reviewed.

Data availability statement Data are available upon reasonable request from the authors. Please contact SS, PhD (sbshah@ucsd.edu).

Open access This is an open access article distributed in accordance with the Creative Commons Attribution Non Commercial (CC BY-NC 4.0) license, which permits others to distribute, remix, adapt, build upon this work non-commercially, and license their derivative works on different terms, provided the original work is properly cited, an indication of whether changes were made, and the use is noncommercial. See: http://creativecommons.org/licenses/by-nc/4.0/.

\section{ORCID iDs}

Brian M Ilfeld http://orcid.org/0000-0002-6144-3273

Rodney A Gabriel http://orcid.org/0000-0003-4443-0021

\section{REFERENCES}

1 Iffeld BM. Continuous peripheral nerve blocks: an update of the published evidence and comparison with novel, alternative analgesic modalities. Anesth Analg 2017;124:308-35.

2 Myers RR, Powell HC, Heckman HM, et al. Biophysical and pathological effects of cryogenic nerve lesion. Ann Neurol 1981;10:478-85.

3 Trojaborg W. Rate of recovery in motor and sensory fibres of the radial nerve: clinical and electrophysiological aspects. Journal of Neurology, Neurosurgery \& Psychiatry 1970;33:625-38.

4 Trescot AM. Cryoanalgesia in interventional pain management. Pain Physician 2003;6:345-60.

5 Gage AA. History of cryosurgery. Semin Surg Oncol 1998;14:99-109.

6 Campos NA, Chiles JH, Plunkett AR. Ultrasound-guided cryoablation of genitofemoral nerve for chronic inguinal pain. Pain Physician 2009;12:997-1000.

7 Joshi DH, Thawait GK, Del Grande F, et al. MRI-guided cryoablation of the posterior femoral cutaneous nerve for the treatment of neuropathy-mediated sitting pain. Skeletal Radiol 2017:46:983-7.

8 Prologo JD, Lin RC, Williams R, et al. Percutaneous CT-guided cryoablation for the treatment of refractory pudendal neuralgia. Skeletal Radiol 2015;44:709-14.

9 Gabriel RA, Finneran JJ, Trescot AM, et al. Ultrasound-Guided percutaneous Cryoneurolysis for postoperative analgesia after limb amputation. A \& A Practice 2019;12:231-4.

10 Ilfeld BM, Gabriel RA, Trescot AM. Ultrasound-guided percutaneous cryoneurolysis providing postoperative analgesia lasting many weeks following a single administration: a replacement for continuous peripheral nerve blocks?: a case report. Korean J Anesthesiol 2017;70:567-70.

11 Dasa V, Lensing G, Parsons $\mathrm{M}$, et al. Percutaneous freezing of sensory nerves prior to total knee arthroplasty. Knee 2016;23:523-8.

12 Barnard D. The effects of extreme cold on sensory nerves. Ann R Coll Surg Engl 1980;62:180-7.

13 Willenbring S, DeLeo JA, Coombs DW. Sciatic cryoneurolysis in rats: a model of sympathetically independent pain. Part 2: adrenergic pharmacology. Anesth Analg 1995;81:549-54.

14 Willenbring S, Beauprie IG, DeLeo JA. Sciatic cryoneurolysis in rats: a model of sympathetically independent pain. Part 1: effects of sympathectomy. Anesth Analg 1995;81:544-8.

15 Popken $\mathrm{F}$, Land $\mathrm{M}$, Erberich $\mathrm{H}$, et al. The use of a new miniature cryoprobe for ablation of bone tissue: in vivo assessment of the probe and application of the method to bone in a sheep model. BMC Surg 2003;3:3.
16 Jiang J, Goel R, Schmechel S, et al. Pre-conditioning cryosurgery: cellular and molecular mechanisms and dynamics of TNF- $\alpha$ enhanced cryotherapy in an in vivo prostate cancer model system. Cryobiology 2010;61:280-8.

17 Gabriel RA, Finneran JJ, Asokan D, et al. Ultrasound-guided percutaneous Cryoneurolysis for acute pain management: a case report. A A Case Rep 2017:9:129-32.

18 Kim PS, Ferrante FM. Cryoanalgesia: a novel treatment for hip adductor spasticity and obturator neuralgia. Anesthesiology 1998:89:534-6.

19 Apfelbaum JL, Chen C, Mehta SS, et al. Postoperative pain experience: results from a national survey suggest postoperative pain continues to be undermanaged. Anesth Analg 2003;97:534-40.

20 Brummett CM, Waljee JF, Goesling J, et al. New persistent opioid use after minor and major surgical procedures in US adults. JAMA Surg 2017;152:e170504.

21 Iffeld BM, Gabriel RA, Trescot AM. Ultrasound-guided percutaneous cryoneurolysis for treatment of acute pain: could cryoanalgesia replace continuous peripheral nerve blocks? Br J Anaesth 2017;119:709-12.

22 Peters D, Barash IA, Burdi M, et al. Asynchronous functional, cellular and transcriptional changes after a bout of eccentric exercise in the rat. J Physiol 2003:553:947-57.

23 Ward SR, Minamoto VB, Suzuki KP, et al. Recovery of rat muscle size but not function more than 1 year after a single botulinum toxin injection. Muscle Nerve 2018;57:435-41.

24 Detloff MR, Clark LM, Hutchinson KJ, et al. Validity of acute and chronic tactile sensory testing after spinal cord injury in rats. Exp Neurol 2010;225:366-76.

25 Chaplan SR, Bach FW, Pogrel JW, et al. Quantitative assessment of tactile allodynia in the rat paw. J Neurosci Methods 1994;53:55-63.

26 Howarth HM, Alaziz T, Nicolds B, et al. Redistribution of nerve strain enables end-toend repair under tension without inhibiting nerve regeneration. Neural Regen Res 2019:14:1280-8.

27 Whittaker DK. Ice crystals formed in tissues during cryosurgery. I. Light microscopy. Cryobiology 1974;11:192-201.

28 Whittaker DK. Ice crystals formed in tissue during cryosurgery. II. Electron microscopy. Cryobiology 1974;11:202-17.

29 Wagner R, DeLeo JA, Heckman HM, et al. Peripheral nerve pathology following sciatic Cryoneurolysis: relationship to neuropathic behaviors in the rat. Exp Neurol 1995; 133:256-64

30 Wagner R, DeLeo JA, Coombs DW, et al. Spinal dynorphin immunoreactivity increases bilaterally in a neuropathic pain model. Brain Res 1993;629:323-6.

31 Wagner R, DeLeo JA, Coombs DW. Gender differences in autotomy following sciatic cryoneurolysis in the rat. Physiol Behav 1995;58:37-41.

32 Wagner R, Deleo JA. Pre-emptive dynorphin and N-methyl-D-aspartate glutamate receptor antagonism alters spinal immunocytochemistry but not allodynia following complete peripheral nerve injury. Neuroscience 1996;72:527-34.

33 DeLeo JA, Coombs DW, Willenbring S, et al. Characterization of a neuropathic pain model: sciatic cryoneurolysis in the rat. Pain 1994;56:9-16.

34 Willenbring S, DeLeo JA, Coombs DW. Differential behavioral outcomes in the sciatic cryoneurolysis model of neuropathic pain in rats. Pain 1994;58:135-40.

35 Hsu M, Stevenson FF. Wallerian degeneration and recovery of motor nerves after multiple focused cold therapies. Muscle Nerve 2015;51:268-75.

$36 \mathrm{Hsu}$ M, Stevenson F-F. Reduction in muscular motility by selective focused cold therapy: a preclinical study. J Neural Transm 2014;121:15-20.

37 Mori T, Agata N, Itoh Y, et al. Stretch speed-dependent myofiber damage and functional deficits in rat skeletal muscle induced by lengthening contraction. Physiol Rep 2014;2:e12213.

38 Wang DC, Bose P, Parmer R, et al. Chronic intrathecal baclofen treatment and withdrawal: I. Changes in ankle torque and hind limb posture in normal rats. J Neurotrauma 2002;19:875-86.

39 Arguis MJ, Perez J, Martínez G, et al. Contralateral neuropathic pain following a surgical model of unilateral nerve injury in rats. Reg Anesth Pain Med 2008:33:211-6.

40 Won R, Jung SJ, Park YG, et al. Crossed-withdrawal reflex in a rat model of neuropathic pain: implications in neural plasticity. Neurosci Lett 2004;369:239-44.

41 Milligan ED, Twining C, Chacur M, et al. Spinal glia and proinflammatory cytokines mediate mirror-image neuropathic pain in rats. J. Neurosci. 2003;23:1026-40.

42 Koltzenburg M, Wall PD, McMahon SB. Does the right side know what the left is doing? Trends Neurosci 1999;22:122-7.

43 Stuesse SL, Cruce WLR, Lovell JA, et al. Microglial proliferation in the spinal cord of aged rats with a sciatic nerve injury. Neurosci Lett 2000;287:121-4.

44 Rob C, May AG. Neurovascular compression syndromes. Adv Surg 1975;9:211-34.

45 Werner RA, Andary M. Carpal tunnel syndrome: pathophysiology and clinical neurophysiology. Clin Neurophysiol 2002;113:1373-81.

46 Muglia U, Vita G, Laura' R, et al. Morphometric comparison between contralateral sciatic nerves in the male and female rabbit. Anat Histol Embryol 1997:26:147-50.

47 Christensen MB, Tresco PA. Differences exist in the left and right sciatic nerves of naïve rats and cats. Anat Rec 2015:298:1492-501. 